\title{
Hybrid Management of Complicated Endovascular Coil-Dislodgement in the Distal Left Subclavian Artery in a Patient with Complex Aortic Disease
}

\author{
Petra Wellmann ${ }^{1}$, Thomas. Helmberger ${ }^{2}$, Oliver Deutsc ${ }^{1}$, Walter. Eichinger ${ }^{1}$ and \\ Brigitte Gansera $^{1}$ \\ ${ }^{1}$ Department of Cardiovascular Surgery, Klinikum Bogenhausen, Munich, Germany \\ ${ }^{2}$ Department of Radiology, Klinikum Bogenhausen, Munich, Germany
}

Correspondence should be addressed to: Petra Wellmann; petra_well@web.de

Received date: 16 February 2014; Accepted date: 28 May 2014; Published date: 19 August 2015

Academic Editor: Stefan Acosta

Copyright $@$ 2015. Petra Wellmann, Thomas. Helmberger, Oliver Deutsc, Walter. Eichinger and Brigitte Gansera. Distributed under Creative Commons CC-BY 4.0

\begin{abstract}
Stent-graft placement for endovascular aortic repair requires an adequate proximal and distal landing zone in the aorta of at least $2 \mathrm{~cm}$ for save and sufficient anchoring and to avoid early or late endoleaks. Ligation and rerouting of one or more supraaortal vessels can be necessary for a sufficient stent-graft placement. We present the clinical management and outcome of a patient who underwent percutaneous stent therapy after a redo-replacement of the ascending aorta and complex surgery of the aortic arch, compromised by a large aneurysm of the ascending-, the thoracic aorta and the aortic arch. The stent-graft procedure necessitated an occlusion of the left subclavian artery by coils, which was complicated by coil-dislodgement. The retrieving of the dislodged coils could be successfully achieved via the brachial artery using a custom made goose snare.
\end{abstract}

Keywords: EVAR- complicated coil migration

\section{Introduction}

Proximal ligation of one or more supraaortic vessels prior to endovascular stent graft placement is an accepted alternative to major open thoracic surgery in the therapy of complex thoracic aortic disease (Garzon 2005). It enables an adequate graft placement, which is necessary to maintain cerebral perfusion and which is preventing primary retrograde perfusion of the aneurysm sack.

Primary dislodgement or secondary coilmigration represent potentially severe complications of this procedure. Several techniques and devices had been described for the retrieval of the migrated bodies in the literature- yet no standard method is established due to the highly variable

Cite this Article as: Petra Wellmann, Thomas. Helmberger, Oliver Deutsc, Walter. Eichinger and Brigitte Gansera (2015), "Hybrid Management of Complicated Endovascular Coil-Dislodgement in the Distal Left Subclavian Artery in a Patient with Complex Aortic Disease," Journal of Radiology Research and Practice, Vol. 2015 (2015), Article ID 504819, DOI: 10.5171/2015.504819 
anatomical situations (Gottardi 2008, Younes 2010). In this case report we present the clinical management and outcome of a patient with a large aneurysm of the thoracic aorta, who received previous, combined open surgery of the ascending aorta and the aortic arch, and then was treated by aortic- endovasculartherapy complicated by an intra-procedural coil- dislodgement, followed by immediate coil-retrieval.

\section{Case-Presentation}

A 68-year old male patient with a long lasting history of aortic disease, with an isolated aneurysm of the ascending aorta and severe valve insufficiency received replacement of the aortic valve and proximal ascending aorta using a mechanical aortic valve composite graft in February 2012. At a new admission 6 months later he presented an aneurysm of the distal ascending aorta, the aortic arch and the proximal descending aorta (diameter 6 centimeters). In a redooperation we performed a complex surgical therapy namely a prosthetic replacement of the distal ascending aorta, the aortic arch (30 mm Dacron- prosthesis), reimplantation of the brachiocephalic trunk and re-implantation of the left common carotid artery (10 mm Dacron- prosthesis). Four months after uncomplicated surgical redo, an interdisciplinary management of a small leakage at the distal anastomosis within the still present aneurysm-sack of the proximal descending aorta was planned by EVAR, to avoid translocation surgery in the multimorbide patient.

Diameter of the distal descending aorta was only $3 \mathrm{~cm}$. Decision-making against performance of frozen elephant trunk at the time of redo was primarily based on the fact that diameter of distal descending aorta was small, and frozen elephant trunk did not seem not to be necessary.

Decision for stent graft and primarily coiling of the left subclavian artery (SA) was based on anatomical vascular situation. Rationale for coil occlusion avoiding retrograde perfusion of the aneurysm sack would have been a "planned" type I endoleak. Primarily 5 coils (diameter 8-10 mm/ $20 \mathrm{~cm}$ length) were uncomplicatedly placed. The reason for using coils instead of vascular occluder, e.g. "amplatzer vascular plug", was due to economic considerations.

Uncomplicated stent graft placement (34mm diameter, $150 \mathrm{~mm}$ length) was performed via a right femoral access route using a 24-French-sheat Stent-graft-System (Valiant (R), Medtronic, Meerbusch am Niederrhein, Germany). With a safety distance to the left carotid artery, length of the landing-zone for the stent graft was $25 \mathrm{~mm}$ within the primarily implanted $30 \mathrm{~mm}$ Dacron prosthesis.

However, at the moment of unfolding the graft- most likely due to changes of blood pressure and flow, the aortic flow was rerouted into the SA pushing the coils distally into the axillary artery.

Unfortunately, no methods for reducing the blood flow, like inflow occlusion or rapid pacing, were performed. Clinically a "coolness" of the left arm was noticed, and fluoroscopy and angiography confirmed the coil package within the axillary artery (Fig.1 and Fig. 2). To prevent critical limb ischemia and to avoid secondary damage of the subclavian and axillary artery; a combined approach for the coil retrievement was made: transarterial catching of the coils and removal out of the artery via an arteriotomy. After puncture of the left cubital artery, a $6 \mathrm{~F}$ sheat was introduced at a hand made goose snare $4 \mathrm{~F}$ cobra catheter together with a 0.0014 inch guide wire) and placed into the SA passing the coil package. By pulling back the goose snare, the coil package could luckily be catched and retrieved to the puncture site and easily removed out of the artery by a small arterial incision. Rationale for using a "handmade" goose snare and not one of the commercially available normal goosesnares were the costs of such handmade goose snares, which are about 80 percent lower than that of the commercial made ones. The required and cheap material for such a simple "hand-made goose snare" consists of only a $300 \mathrm{~cm}$ length guidewire and a 4 French catheter. The subsequent 
intraoperative angiography revealed a patent flow within the subclavian-, axillaryand peripheral arm arteries. Finally, after finishing the entire procedure, postoperative angiography and computed tomography 3 days after the intervention documented mostly complete thrombosis of the origin of the subclavian artery, collateralization via retrograde flow from the vertebral artery and no stent endoleak. (Fig.3).

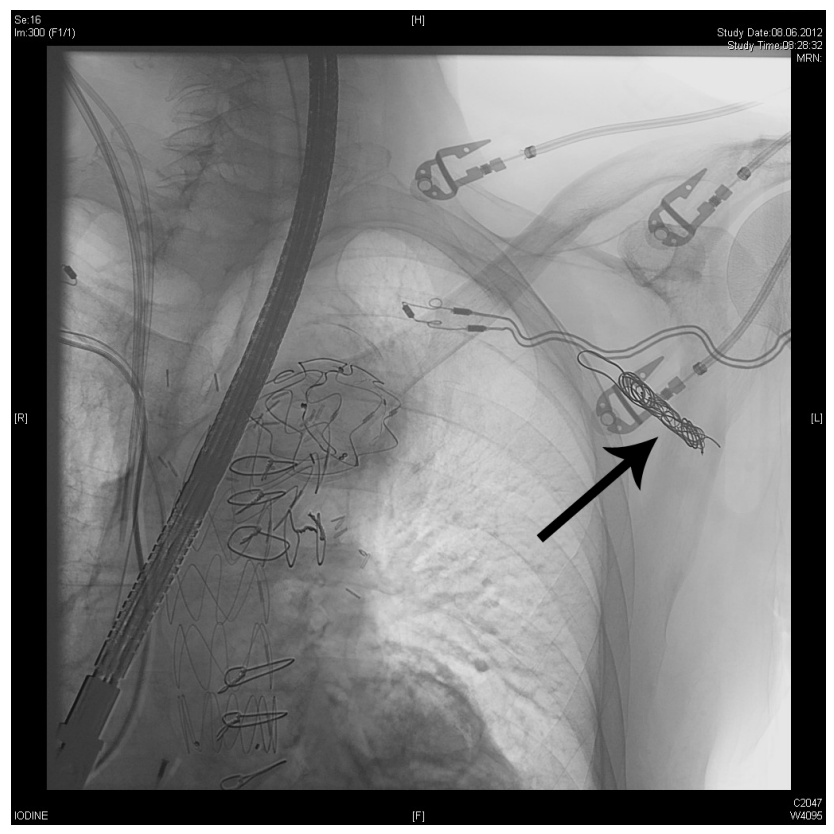

Fig.1: Primarily Correct Position of the Coils (Arrow) in the Subclavian Artery

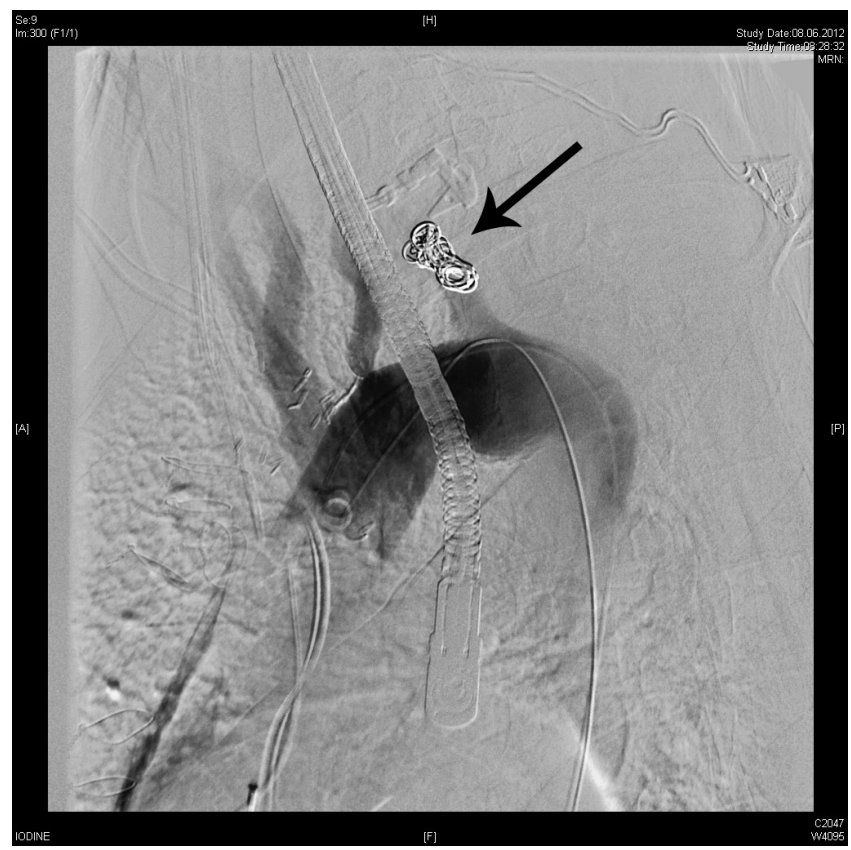

Fig. 2: Angiography after Implantation of the Stent-Graft (Valiant ${ }^{\mathrm{R}}$ ) and Coil Migration (Arrow) 


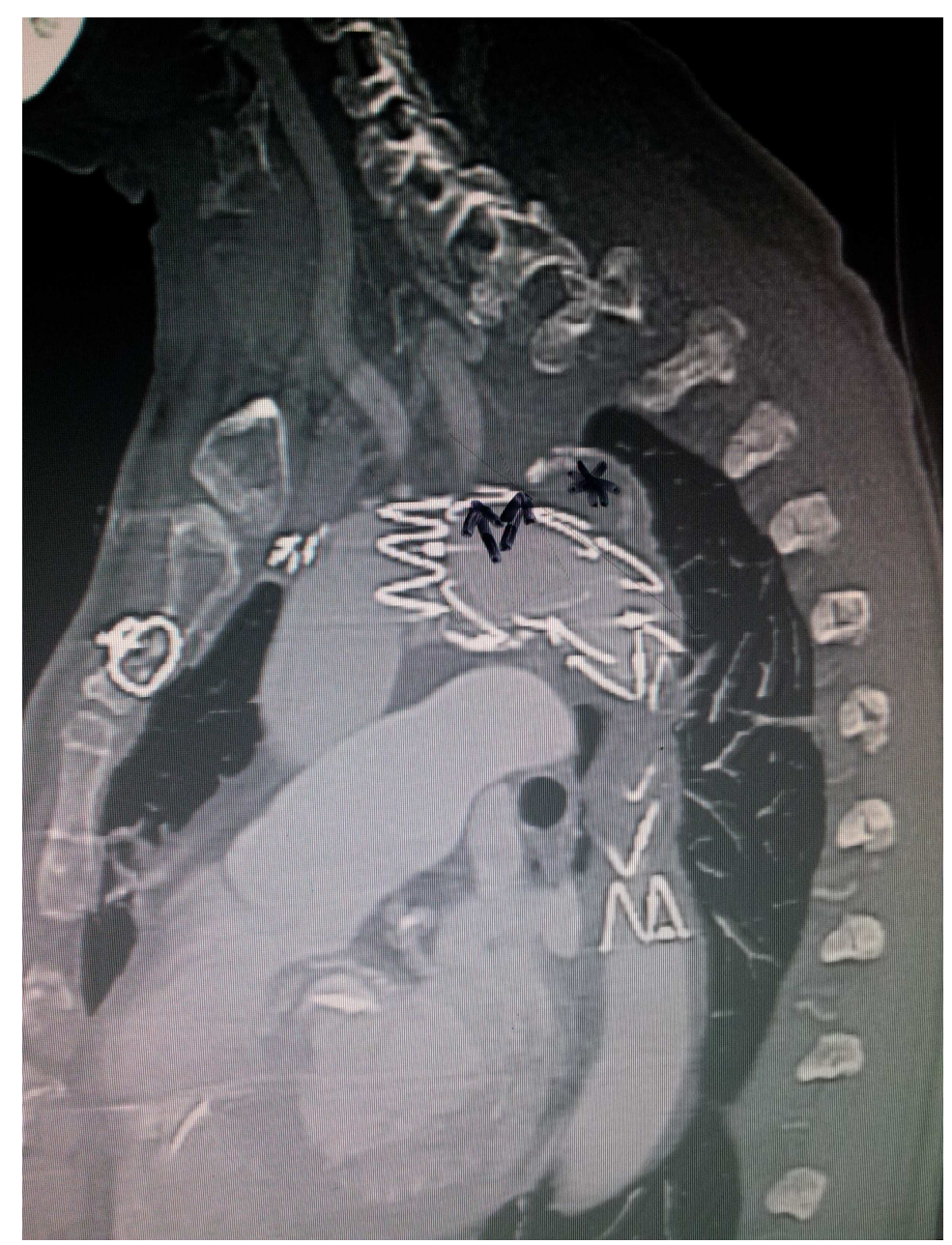

Fig.3: Computed Tomography 3 Days after Stent- Graft Implantation. No Endoleak (Arrow). Excluded Sack Aneurysm (Star).

\section{Discussion}

Endovascular occlusion of the subclavian artery has been reported to be successfully performed in cases of intentional left subclavian artery coverage directly by the endograft (Peterson 2008, Meyer 2009). Furthermore, retrograde aneurysm sack perfusion by reverse flow of the SA is also reported.

In many centres, therefore a preventive occlusion procedure is performed simultaneous or prior to the EVAR procedure. Various techniques can be applied for this task such as coiling or placing an endovascular plague. Nevertheless, this procedure- even if it looks rather simple for the interventionalist- can be affected seriously , as described in our case, when the pressure and flow situation in the aortic arch and the subclavian artery are changing significantly.

In retrospect, it would have been smarter placing the stent graft first and controlling the effectiveness of the exclusion of the aneurysm sack. Then, if a retrograde perfusion of the SA would have been detectable, the coils could have been placed. With this approach a centrifugal flowin the SA definitively would have been avoided. The common technical procedure: first stent-graft and then deployment of coils was not performed in this specific case, due to the huge lumen diameter and the broad basis of the SA at her origin that might bear the risk of producing an endoleak. Moreover, the complication might not have 
happened if bigger coils had been used.

Our case report might be a helpful contribution in decision making towards emergent and hopefully successful intervention in the onset of this complication.

\section{Comment}

Coil migration may leads to serious consequences in endovascular aortic surgery, in particular to embolization and consequent ischemia in the area of peripheral vessels. In the present case, the device (custom made goose- snare) provides a secure technique for retrieval of the dislocated coils.

\section{References}

1.Garzon, G., Fernandez-Velilla, M., Marti, M., Acitores, I., Ybanez, F. \& Riera, L. (2005). "Endovascular Stent-Graft Treatment of Thoracic Aortic Disease," Radiographics 25:229-224

2.Gottardi, R., Funovics, M., Eggers, N., Hirner, A., Dorfmeister, M., Holfeld, J., Zimpfer, D., Schoder, M., Donas, K.,
3.Weigang, E., Lammer, J., Grimm, M. \& Czerny, M. (2008). "Supra-aortic Transposition for Combined Vascular and Endovascular Repair of Aortic Arch Pathology," The Annals of Thoracic Surgery 86:1524-1529

4.Meyer, C., Probst, C., Stunk, H., Schiller, W. \& Wilhelm, K. (2009). "SecondGeneration Amplatzer Vascular Plug (AVP) for the Treatment of Subsequent Subclavian Backflow Type II Endoleak after TEVAR," CardioVascular and Interventional Radiology 32: 2164-2167

5.Peterson, M. D., Wheatley, G. H., Kpodonu, J., Williams, J. P., Ramaiah, V. G., Rodriguez-Lopez, J. A. \& Dietrich, E. B. (2008). "Treatment of Type II Endoleaks Associated with Left Subclavian Artery Coverage during Thoracic Aortic Stent Grafting," The Journal of Thoracic and Cardiovascular Surgery 136: 1193-1199

6.Younes, H. K., Davies, M. G., Bismuth, J., Naoum, J. J., Peden, E. K., Reardon, M. J. \& Lumsden, A. D. (2010). "Hybrid Thoracic Endovascular Aortic Repair: Pushing the Envelope," Journal of Vascular Surgery 51:259-266 